\title{
Following peak profiles during elastic and plastic deformation: A synchrotron-based technique
}

\author{
H. Van Swygenhoven, B. Schmitt, P. M. Derlet, S. Van Petegem, A. Cervellino, \\ Z. Budrovic, S. Brandstetter, A. Bollhalder, and M. Schild \\ Paul Scherrer Institute, CH-5232 Villigen PSI, Switzerland
}

(Received 19 May 2005; accepted 25 November 2005; published online 30 January 2006)

\begin{abstract}
Understanding the elastic and plastic deformation properties of nanostructured metals requires the development of in situ testing methods that can follow the footprints of the deformation mechanism(s) during mechanical testing. Here we present an in situ synchrotron x-ray-diffraction technique which allows the measurement of diffraction profiles continuously during mechanical testing, providing an in situ peak profile analysis capability. The in situ approach is achieved thanks to the development of a microstrip detector allowing the instantaneous measurement of the diffraction pattern over a $2 \theta$ range of $60^{\circ}$. This in situ technique allows for the first time a comparison of the footprints of the plastic deformation mechanism during loading and after unloading. The measurements are performed on several types of freestanding dog bones, covering sample thicknesses down to the submicron range. () 2006 American Institute of Physics.
\end{abstract}

[DOI: $10.1063 / 1.2162453]$

\section{INTRODUCTION}

There is a long-standing interest from a materials science viewpoint to understand size effects in mechanical behavior of metals. It is well known that the strength of metals increases with decreasing grain size, a behavior that is well described by the phenomenological Hall-Petch relation down to grain sizes of $100 \mathrm{~nm}$ and even lower. ${ }^{1,2}$ On the other hand, it has been demonstrated that the strength of a material increases when sample dimensions are reduced to the submicron meter scale. ${ }^{3}$ Deformation mechanisms in coarsegrained metals are based on a dislocation mechanism where dislocations are created during deformation with their propagation and multiplication being an essential mechanism for the resulting ductility and strength. Size effects are currently discussed in terms of either a restriction in dislocation nucleation and/or a restriction in dislocation propagation/ multiplication. Some of the mechanisms proposed do not leave any footprint at all after deformation. ${ }^{4,5}$ Transmission electron microscopy is helpful in this research area, but the method is only useful when the deformation mechanism leaves a postmortem footprint. Furthermore, the method is handicapped by the thinning procedures used for making electron transparent samples, which might alter the footprints of the deformation mechanism. ${ }^{6}$

The above research topic therefore calls for an in situ technique that allows the deformation mechanism during loading to be probed, in such a way that interpretation does not rely solely on what is left over at unloading.

$\mathrm{X}$-ray-diffraction (XRD) profile analysis is a well-known technique for microstructural analysis, where the broadening of the peaks result from limitations in the spatial extent of the coherent-scattering volumes (in our case the grain size) and the presence of inhomogeneous strain. The first type of broadening is diffraction order independent, whereas the sec- ond is order dependent. Possible sources for inhomogeneous strain are lattice dislocations, but other sources can be of equal importance, especially in nanocrystalline structures, such as an excessive number of extrinsic grain-boundary (GB) dislocations due to the presence of unrelaxed GBs and triple junctions, or elastic inhomogeneous strains resulting from elastic anisotropy originating from different strains in different grains and/or strain gradients within grains. Several theories exist to estimate dislocation densities from measured diffraction patterns, but because origins of peak broadening are many, and because most of these theories are developed primarily for dislocation networks, they are not particularly suited to investigating nanocrystalline metals where most of the peak broadening might come from sources other than dislocation networks. ${ }^{7}$ Here we present an in situ synchrotron-based technique allowing the bulk peak profile to be measured during in situ tensile testing of conventional dog-bone samples as a function of the macroscopic stressstrain curve at strain rates typically in the range of $10^{-5}$. The success of the technique relies on the excellent time resolution offered by a developed microstrip detector (currently only available at the Swiss Light Source), covering an angular range of $60^{\circ}$ with an intrinsic angular resolution of $0.004^{\circ}$. In this setup several diffraction peaks can be measured in a few seconds thanks to the high intensity of the source, which can then be read out in a negligible amount of time, allowing to perform time-resolved measurements during both elastic and plastic deformation. Other detection systems are either too slow (scanning analyzer detector) or do not cover the required angular range with a good resolution [charge-coupled devices (CCDs) or other position sensitive detectors].

In the past other techniques have been developed for gathering information from XRD peak profiles on strain distribution in polycrystalline samples, however, with different 
goals. For example, the computer-aided x-ray double-crystal diffraction method, which uses a position sensitive detector to measure rocking curves of single grains, allows for a static mapping of micron level plasticity over a macrosized sample. ${ }^{8,9}$ More recently a synchrotron-based in situ XRD method has been developed for determining the mechanical stress evolution in submicron thin films that are deposited on compliant substrates. ${ }^{10}$ The technique comprises an initial " $\sin ^{2} \psi$ " measurement to establish the absolute stress values followed by periodic " $\sin ^{2} \varphi$ " measurements during straining to determine the stress increments. The measurements are done in Laue transmission and require a noncontinuous loading profile, in which the load is kept constant during measurement. The technique has been successfully further elaborated for following the spatial strain distribution in $\mathrm{Al}$ thin films during tensile deformation. ${ }^{11}$ The technique is, however, not very well suited for following plastic deformation mechanism in bulk nanocrystalline metals because measurements are done in transmission requiring thin samples deposited on a compliant substrate.

Our experimental setup relies on the high flux of a thirdgeneration synchrotron source with tunable energies and on the availability of a unique detector that allows very fast measurement of a powder-diffraction pattern covering an angular range of $60^{\circ} .{ }^{12}$ This detector allows the measurement of peak profiles during continuous loading, avoiding waiting times where the load is kept constant and stress relaxes. The use of a tensile machine with a small strain step increase allows following the behavior of peak position and peak broadening during elastic and plastic deformation.

In this article we first describe the experimental method in detail, including the fast profile analysis method which is necessary to evaluate the tremendous amount of recorded data. Then by means of an illustrative example, the case of nanocrystalline (nc) electrodeposited (ED) $\mathrm{Ni}$ with a mean grain size of $30 \mathrm{~nm}$, we demonstrate the impact of the in situ measurements on the understanding of strain accommodation in polycrystalline metals with grain sizes towards and in the nanometer regime. In a future outlook, we briefly describe the possible extensions of this technique for other investigations such as the application of the technique for the investigation of plastic deformation mechanism in freestanding thin films.

\section{EXPERIMENTAL PROCEDURES AND DATA ANALYSIS}

\section{A. Experimental setup}

A schematic drawing of the in situ deformation apparatus is shown in Fig. 1. Here a monochromatic focused x-ray beam illuminates a specimen mounted in a tensile machine that is mounted on a goniometer. A CCD camera is used to observe the specimen during deformation. The diffracted $\mathrm{x}$-ray beam, measured in a reflective geometry, is recorded using a unique microstrip detector that allows the recording of a complete diffraction spectrum in just a few seconds. In what follows we describe the individual components in more detail.

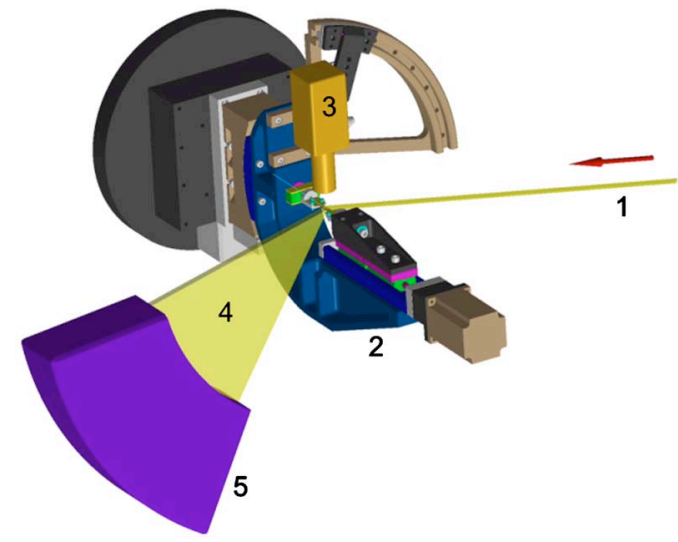

FIG. 1. (Color online) Schematic drawing of the in situ deformation apparatus showing (1) the incoming $\mathrm{x}$-ray beam, (2) the tensile machine mounted on the goniometer, (3) the CCD camera used to observe the sample during deformation, (4) the diffracted beam, and (5) the microstrip detector.

\section{B. X-ray source}

All measurements were performed at the powderdiffraction station of the materials science (MS) beamline at the Swiss Light Source (SLS, Villigen, Switzerland) since it is currently the only beamline equipped with the required detector. The SLS is a third-generation light source, operated at $2.4 \mathrm{GeV}$ with an electron-beam current of up to $400 \mathrm{~mA}$. The MS beamline produces a high flux of hard $\mathrm{x}$ rays from a wiggler source. The beamline optics consists of two Rhcoated Si mirrors and a double-crystal Si (111) monochromator. A monochromatic x-ray beam with energies between 5 and $40 \mathrm{keV}$, and a spot size of about $150 \times 450 \mu \mathrm{m}^{2}$, can be produced. Further reduction of the beam's final cross section is achieved by using horizontal and vertical slits. The distance between the central part of the wiggler and the powder-diffraction station is about $36 \mathrm{~m}$. The main parameters of the MS beamline are summarized in Table I and further details can be found in Ref. 13.

\section{Microtensile machine}

The microtensile machine (MTM) is mounted on the goniometer with the tensile axis parallel to the goniometer axis. Thus the incoming beam is perpendicular to the tensile axis, however, its incident angle relative to one of the sample's surfaces can be chosen and kept constant during the experiment. The displacement actuator system running the linear stage is a spindle system with spherical bearings for transferring the rotational motion of a stepper motor into linear motion, while ensuring the motion is continuous with a minimum step size of $16 \mathrm{~nm}$. Driving velocities up to $0.16 \mathrm{~mm} / \mathrm{s}$ can be achieved. The strain is measured by a CCD camera in combination with image recognition software (Messphysik CCD Videoextensiometer ME46). The strain resolution for a

TABLE I. Main operational parameters of the materials science beamline.

Photon energy range

Photon energy resolution

Max flux at $17.5 \mathrm{keV}$

Accepted divergence

$$
\begin{aligned}
& 5-40 \mathrm{keV} \\
& \sim 10^{-4} \\
& 4 \times 10^{12} \mathrm{ph} / \mathrm{s} \\
& 0.23 \times 2.5 \mathrm{mrad}^{2}
\end{aligned}
$$


TABLE II. Load cell specifications.

\begin{tabular}{lccr}
\hline \hline Manufacturer & Type & $\begin{array}{c}\text { Maximum load } \\
(\mathrm{N})\end{array}$ & $\begin{array}{r}\text { Resolution } \\
(\mathrm{mN})\end{array}$ \\
\hline Cooper instruments & LFS 270 & 1 & 0.08 \\
Transducer techniques & MLP 50 & 222 & 12.26 \\
Transducer techniques & MLP 300 & 1334 & 62.19 \\
\hline \hline
\end{tabular}

typical measuring view field of $5 \mathrm{~mm}$ is about $\sim 100 \mathrm{~nm}$. Three types of load cells can be mounted, depending on the required resolution and force range. Their specifications are listed in Table II. These load cells can be used both in tensile and compression.

The MTM is designed to run in three different modes: (a) tensile test mode with a constant driving velocity (constant strain rate), (b) the creep test mode, where the force is kept constant and strain is recorded as a function of time, and (c) strain rate jump test mode, where the strain rate can be changed instantaneously during the course of the measurement.

\section{Samples}

Figure 2 displays scanning electron microscopy (SEM) images of three different types of sample geometry that have been tested. Figure 2(A) shows two freestanding dog bones where the larger dog bone has a total length of $20 \mathrm{~mm}$, a gauge length of $2.75 \mathrm{~mm}$, and a gauge cross section of $1.25 \times 0.2 \mathrm{~mm}^{2}$. The mini-dog-bone has a length of $3 \mathrm{~mm}$, a gauge length of $1.7 \mathrm{~mm}$, and a gauge cross section of 0.2 $\times 0.2 \mathrm{~mm}^{2}$. Both of these samples are cut by wire electrodischarge and then mechanically and electrochemically polished. During deformation the monochromatic X-ray beam is focused onto the central part of the gauge, illuminating a section of less than $500 \mu \mathrm{m}$. Figure 2(B) shows a freestanding polycrystalline Si film dog bone with a gauge cross section of $3.5 \times 600 \mu \mathrm{m}^{2}$. These type of dog bones are produced by surface and bulk micromachining techniques, for more details we refer to Ref. 14. Such geometries can also be used for studying submicron thin films synthesized by magnetron sputtering. ${ }^{15}$

The MTM is equipped with different types of sample grips allowing the mounting of different sample geometries, as shown in Fig. 3. The large dog bone is clamped between two hardened steel plates allowing forces of up to $1 \mathrm{kN}$. The mini-dog-bone sample bow-tie shape is designed to fit into a mating grip made of tungsten carbide [see inset of Fig. 3(B)]; this self-aligning technique requires no clamping or gluing of the specimen. The thin-film Si sample is mounted by gluing the $\mathrm{Si}$ support frame onto the grips. The Si support strips of the frame are then cut prior to testing.
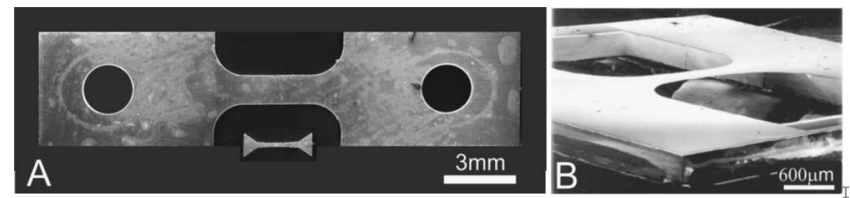

FIG. 2. SEM image of (A) a large dog bone and a mini-dog-bone and (B) the thin-film geometry.

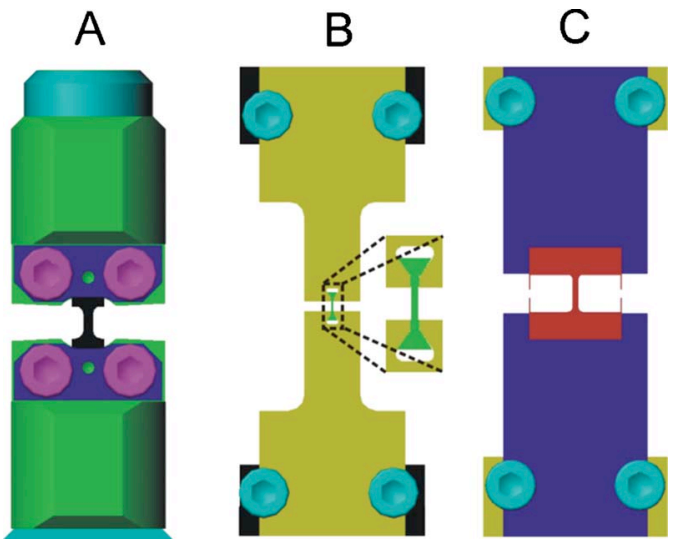

FIG. 3. (Color online) Drawing of the grips used for (A) the large dog bone (colored black), (B) mini-dog bone (colored green), and (C) thin-film dog bone (colored red).

\section{E. Sample environment}

In addition to room-temperature measurements the MTM is also operated at low temperatures. Two cooling devices were used for the low-temperature setup. A CryoJet $\AA$ from Oxford Instruments blows cold nitrogen through a two chamber system constructed from Kapton foil surrounding the specimen and, additionally, a liquid-nitrogen-cooled copper block mounted on one sample grip to minimize heat transfer. The two chamber system was used to prevent ice formation on the specimen. During in situ deformation in the beam, a direct temperature measurement with a thermocouple on the gauge section of the dog bone was not possible, therefore the temperature was calibrated ex situ by comparing the temperature measured by thermocouples on the gauge, on the ears of the sample, and the specimen grips. Within this calibration it could be shown that the temperature gradient along the gauge is less than $5 \mathrm{~K}$, allowing temperature measurement of the specimen during in situ measurement via the temperature measurement on the grips. With this setup a constant temperature of $180 \mathrm{~K}$ at the gauge could be reached for several hours without the formation of ice. To verify the temperature increase due to the beam the temperature was measured with and without beam using a thermocouple on the sample, showing an increase in the temperature of only $0.7^{\circ} \mathrm{C}$ for the duration of the measurements.

\section{F. Microstrip detector}

The microstrip system for time-resolved experiment (MYTHEN) detector system is a solid-state detector specifically designed for time-resolved and fast measurements. It is a modular system covering $60^{\circ}$ in $2 \theta$ using 12 modules. Each module has 1280 channels giving a total of 15360 channels for the entire MYTHEN detector. The detecting element on each module is a 300- $\mu$ m-thick silicon sensor with $8-\mathrm{mm}$ long strips at a pitch of $50 \mu \mathrm{m}$. Each strip is wire bonded to a channel of a specifically developed readout chip. The chip contains 128 channels, each of them having a charge sensitive preamplifier, two shapers as gain stages, a comparator, and a 21 bit counter. The count rate of a channel is about 1 $\mathrm{MHz}$ as measured with pulses. The channels can all count independently and in parallel. The detector is gatable and 
A)

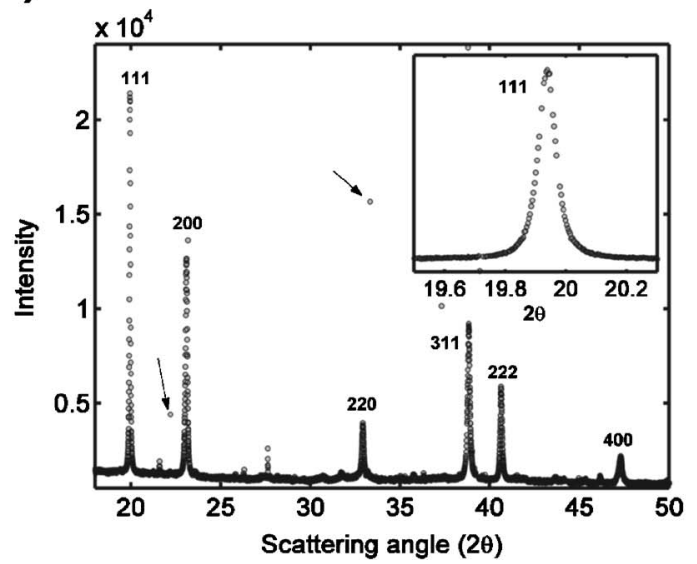

B)

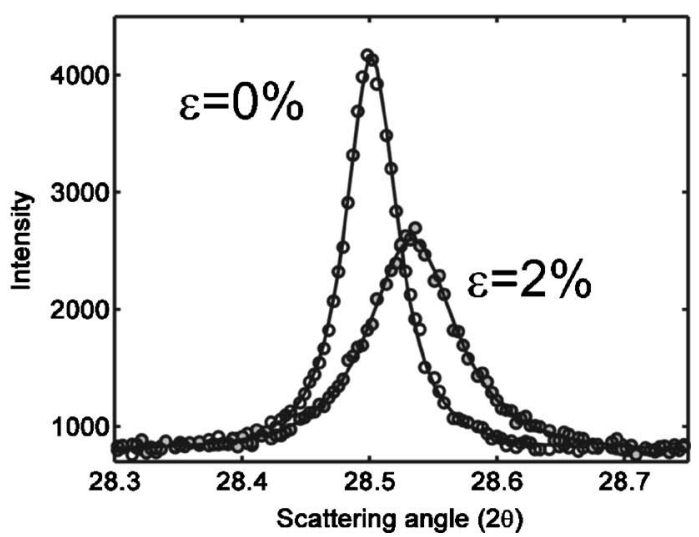

FIG. 4. X-ray-diffraction spectra accumulated by the microstrip detector in $10 \mathrm{~s}$. (A) ED Ni [inset shows a blow up of the (111) peak] and (B) (220) diffraction peak of a freestanding Al film with a thickness of $380 \mathrm{~nm}$. The spectra are taken before deformation and at a tensile strain of $2 \%$, demonstrating peak shift and peak broadening during tensile deformation.

acquisition times can freely be chosen above 100 ns. The readout time of the entire detector is about $250 \mu$ s adding no significant dead time to the measurement. More details can be found in Ref. 12.

Figures 4(A) and 4(B) show typical diffraction spectra accumulated by the microstrip detector over $10 \mathrm{~s}$. Figure 4(A) shows the diffraction spectrum of a small dog bone of ED Ni whereas Fig. 4(B) displays diffraction spectra (before deformation and at a true strain of $2 \%$ ) of a freestanding nc Al film with a geometry similar to that shown in Fig. 2(B) and a film thickness of $380 \mathrm{~nm}$. All measurement points are recorded simultaneously by different channels. A problem in the current version of the readout chip is a missing pulse width defining element between the comparator and the counter. If the pulse width after the comparator or the time between two pulses is too short the countervalue can potentially be lost and the counter can take a random value. This happened for the two points indicated by the arrows in Fig. 4(A).

\section{G. Data analysis}

The count rate of all the channels needs first to be normalized because the count rate varies from channel to channel due to variations in the gain and the comparator threshold. This is done by a so-called flat field correction where normalization factors are calculated for each channel using data from a uniform illumination of the detector. This uniform illumination can be easily achieved using fluorescent light emitted by a conveniently chosen sample. In our case, where $17.5 \mathrm{keV}$ photons are normally used, a Mo salt dissolved in water is used.

The next step in the data analysis is the calculation of the angle for each channel and the elimination of dead or hot channels. Of course one may eliminate hot channels "by eye" but the huge volume of data supports the need for an automated procedure. A significant number of these spurious data points can be removed via an upper and lower intensity thresholds, and in addition the robust-least-squares procedure used to extract peak profiles (described below) will itera- tively deweight, and in some cases eventually remove, any remaining hot channels whose intensities are within the upper and lower global thresholds.

The diffraction peaks could be well fitted using the asymmetric Pearson VII (Ref. 16) given by

$$
\begin{aligned}
P(\theta)= & H\left[1+\left(2^{1 / M_{R}}-1\right)\left(10^{\alpha}+1\right)^{2}\left(\frac{\theta-\theta_{0}}{w}\right)^{2}\right]^{-M_{R}} \\
& \text { if } \theta \geqslant \theta_{0}, \\
P(\theta)= & H\left[1+\left(2^{1 / M_{L}}-1\right)\left(10^{-\alpha}+1\right)^{2}\left(\frac{\theta-\theta_{0}}{w}\right)^{2}\right]^{-M_{L}} \\
& \text { if } \theta<\theta_{0},
\end{aligned}
$$

where $\theta$ is the diffraction angle, $\theta_{0}$ the peak maximum, $H$ the height, $w$ is the full width at half maximum (FWHM), $\alpha$ the (logarithmic) asymmetry parameter, and $M_{L}$ and $M_{R}$, respectively, the left and right decay exponents. For high values of the latter, the Pearson VII tends to a Gaussian, while for $M$ $=1$ it tends to a Cauchy (Lorentzian) profile. An example of such a fit is given in Fig. 5. In general the diffraction peaks of nc Ni are fairly symmetric with $M$ values ranging between 1 and 4.

For the actual fitting of a spectrum a least-squares objective function is constructed from the residual intensities of each channel,

$$
\chi=\sum_{i}\left(\frac{r_{i}}{\sqrt{I_{i}}}\right)^{2}
$$

where the residual is defined as

$$
r_{i}=I_{i}-P_{i}-b g_{i} .
$$

Here $I_{i}$ is the intensity at two-theta angle $\theta_{i}, P_{i}$ is the value of the Pearson VII fit at $\theta_{i}$, and $b g_{i}$ is the value of the background fit at $\theta_{i}$. The background function is an $n$th order polynomial. A robust-least-squares algorithm is used to iteratively perform a reweighted least-squares minimization of the objective function using the following procedure. ${ }^{17}$

(1) Fit the model by an unweighted least squares (that is, $\chi$ ). 

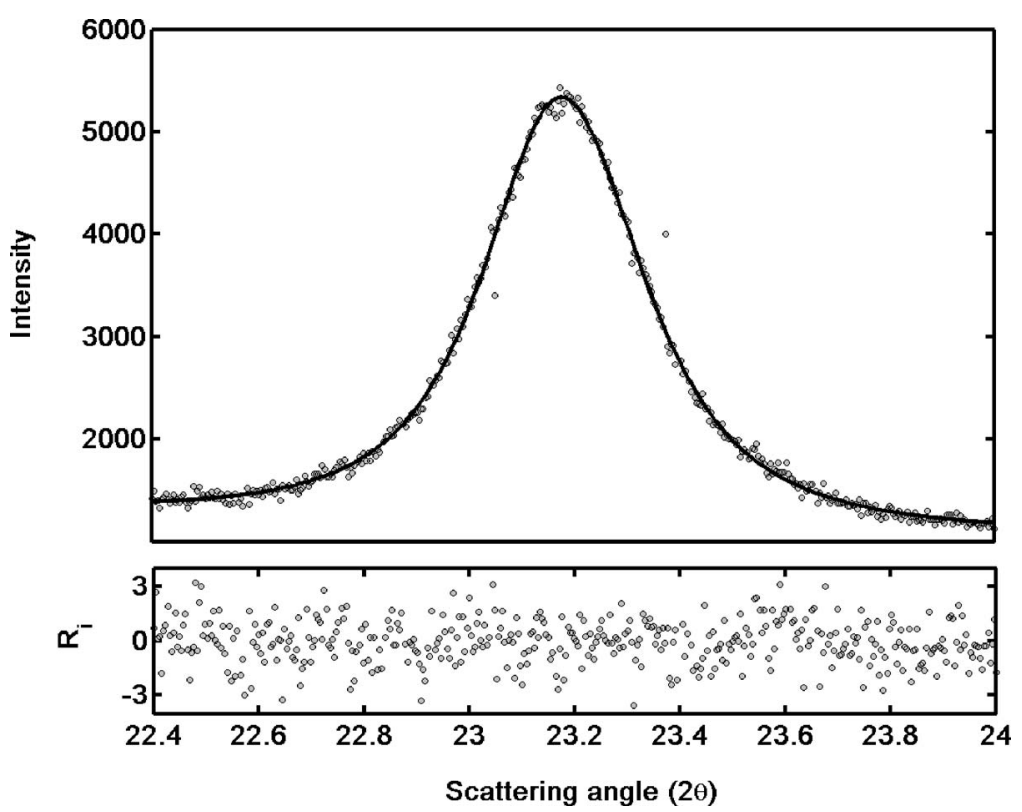

FIG. 5. A typical (200) peak fitted using a Pearson VII function. Below the residuals of the converged fit are shown.

(2) Calculate the standardized residuals, $u_{i}=r_{i} / K s$, where $K$ is a tuning constant equal to 4.685 and $s$ is the robust variance given by MAD/0.6745 (MAD is the median absolute deviation of the residuals, see Ref. 17 for more details).

(3) Compute the robust weights via $w_{i}=\left(1-u_{i}^{2}\right)^{2}$ if $\left|u_{i}\right|<1$ (i.e., if $r_{i}<K s$ ) and $w_{i}=0$ otherwise.

(4) Redo the fit using the weighted minimizer: $\chi$ $=\sum_{i} w_{i}\left(r_{i} / \sqrt{I_{i}}\right)^{2}$.

(5) Repeat steps 2 to 4 until the MAD changes by no more than a user selected fractional tolerance.

Figure 5 also displays the standardized residuals of the converged Pearson VII fit to a 200 peak. The good quality of the fit is evident because no residual is significantly larger than three standard deviations. Note also that the two hot channels in the raw data have converged residuals weights of zero and therefore are automatically excluded from the final converged fit (as evidenced in the displayed residuals). This feature of the robust-least-squares procedure is also found to be useful when additional narrow peaks arising from the grips or ice are present in the spectra: the associated weights progressively approaching zero in the neighborhood of the peak thereby reducing its effect on the final converged Pearson VII fit of the much broader peak originating from the deforming dog bone. For additional peaks that have a width approaching that of the deforming sample peaks, additional Pearson VII functions must be explicitly included in the fitting procedure.

\section{IN SITU SCATTERING GEOMETRY}

\section{A. Sample considerations}

The main difference between this experiment and a conventional $\theta / 2 \theta$ x-ray-diffraction experiment is the fact that the angle between the incoming $\mathrm{x}$-ray beam and the sample remains fixed during the complete tensile experiment. This has important consequences for the types of samples that can be investigated and for a correct interpretation of the results. Figure 6 details a simplified schematic representation of the

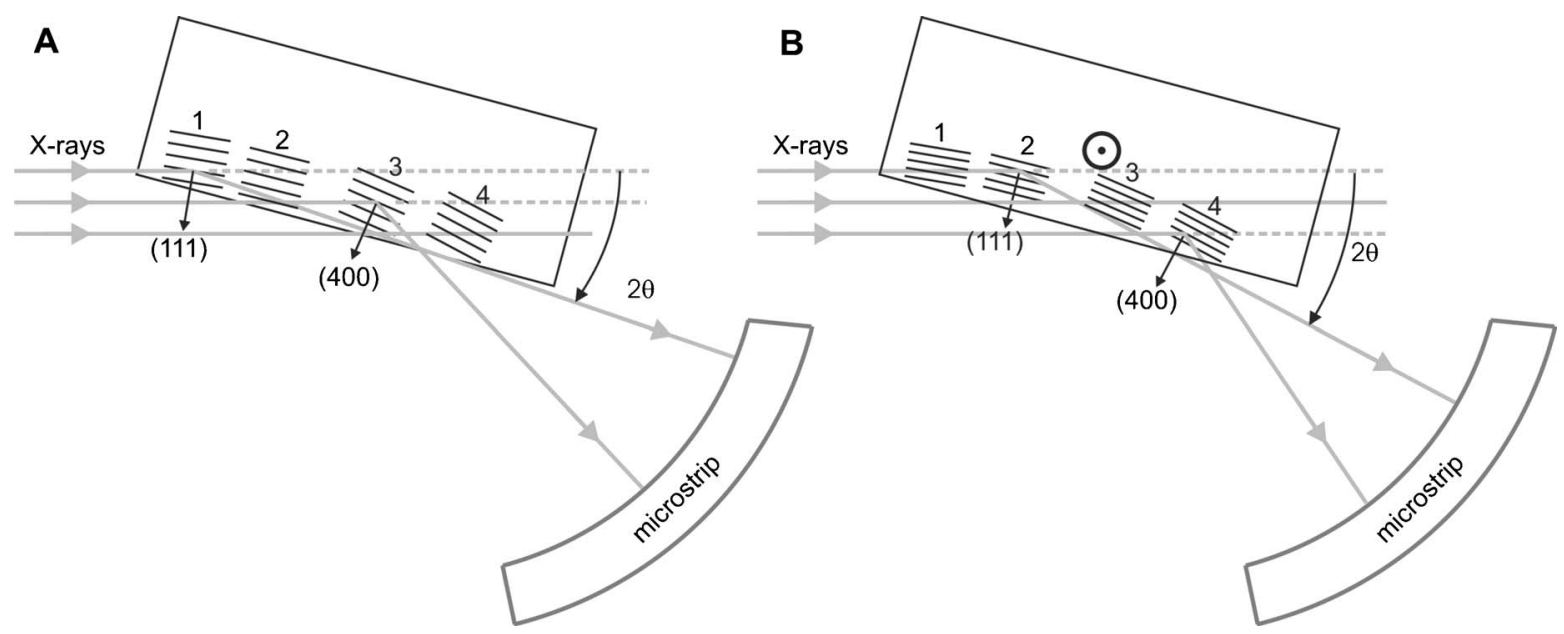

FIG. 6. Scattering geometry for x-ray diffraction with fixed incoming angle: (A) before deformation and (B) under tensile loading conditions, the tension is applied perpendicular to the drawing plane. Note that the microstrip detector is not drawn on scale. 


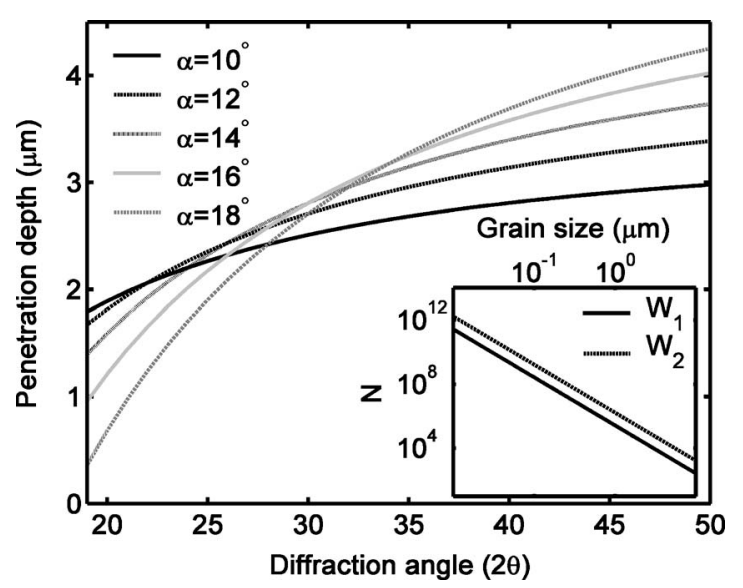

FIG. 7. Penetration depth in Ni as function of diffraction angle for several values of $\alpha$ (the angle between the incoming beam and the sample). The inset shows the number of grains contributing to the (220) peak in case of a mini-dog-bone $\left(W_{1}=200 \mu \mathrm{m}\right)$ and a large dog bone $\left(W_{2}=1250 \mu \mathrm{m}\right)$.

typical scattering geometry for a polycrystalline sample. In Fig. 6(A) the x-ray beam illuminates an undeformed sample under a fixed angle, where the assumption of a parallel beam is justified given the small divergence of the beam (see Table I). In this figure only grains 1 and 3 have the correct orientation to fulfill the Bragg diffraction condition and therefore contribute to the diffraction peaks that are measured by the multistrip detector. It is important to note that different grains contribute to different measured diffraction peaks. Figure now displays schematically the diffraction geometry under tensile loading conditions where the tensile axis is perpendicular to the viewing plane. Due to the Poisson response of the material, the $\mathrm{x}$ rays see a reduced lattice spacing and as a consequence grains 1 and 3 are no longer in the correct orientation to contribute to the $\mathrm{x}$-ray spectrum. On the other hand, it is now grains 2 and 4 that are in the correct orientation to fulfill the Bragg diffraction condition and be measured by the multistrip detector. In such an experiment, the reduced lattice spacing due to the tensile loading shifts the diffraction peaks towards larger angles.

As a consequence a large amount of grains must be present in the sample in order to have sufficient grains in diffraction conditions at all times during deformation. We note that because of the scattering geometry and twodimensional detector position, only grains containing a diffracting plane normal orthogonal to the tensile axis will contribute to the spectrum. Due to these geometrical constraints, the measured relative intensities have to be taken with care, especially when texture is present or the sample contains large grains. For a sample with texture, a texture analysis unique to the present scattering geometry has to be performed. ${ }^{18}$

The number of diffracting grains can be estimated as follows. First we calculate the illuminated volume. The illuminated volume is given by $V=\tau \times W \times 500 \mu \mathrm{m}$ with $W$ the width of the dog bone and $\tau$ the penetration depth. The attenuation of the $\mathrm{x}$ rays can be described by an exponential attenuation law with a given attenuation coefficient. The penetration depth is then given by ${ }^{19}$

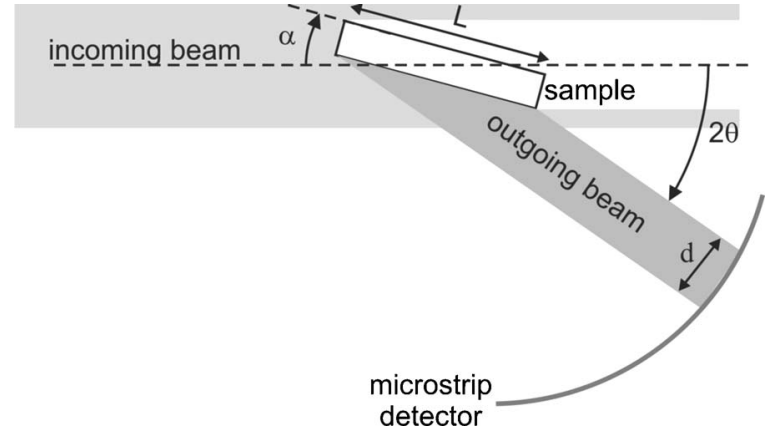

FIG. 8. A schematic drawing of influence of the sample geometry on the resolution function. The tensile axis is perpendicular to the viewing plane.

$$
\tau=\frac{1}{\mu k} \text { with } k=\frac{\sin \alpha+\sin (2 \theta-\alpha)}{\sin \alpha \sin (2 \theta-\alpha)},
$$

where $k$ is a geometrical factor describing the influence of the attenuation of the $\mathrm{x}$-ray beam as function of the angle between the incoming beam and the sample $(\alpha)$, and the diffraction angle $(2 \theta)$. This is demonstrated in Fig. 7 for the case of $\mathrm{Ni}\left[\mu=459 \mathrm{~cm}^{-1}\right.$ at $17.5 \mathrm{keV}$ (Ref. 20)] for several values of $\alpha$. With increasing $\alpha$ the variations of the penetration depth as function of the diffraction angle strongly increase favoring the use of a small incoming angle. However, the number of photons hitting the sample is proportional to $\sin \alpha$. Therefore measurements are usually performed at $\alpha=15^{\circ}$.

Only a particular fraction of illuminated grains will contribute to the measured diffraction peak. Already Ladell ${ }^{21}$ presents a detailed analysis of the powder-diffraction experimental factors, including the normalization of the observed intensity to the fraction of diffracting grains which are seen by the detector. Noyan and Caldor ${ }^{22}$ refine this calculation for the very sensitive microbeam case, where they evaluate the number of grains as a function of beam divergence by assuming that the intrinsic width of the Bragg peak is negligible in comparison with the beam divergence. On the SLS instrument, when measuring nanocrystalline samples, the situation turns out to be the opposite; the beam divergence is negligible in comparison with the intrinsic Bragg peak width. We therefore present an analogous order-of-magnitude calculation unique to the present scattering and nanocrystalline sample geometries. The calculation is presented in detail in the Appendix and results in the fraction of grains that contribute to a particular Bragg peak being given by

$$
\frac{d N}{N} \approx \frac{0.028 p H / L}{\cos \theta_{h}},
$$

where $p$ is the multiplicity of the Bragg peak $h$ being considered, $H$ is the width of the multistrip detector orthogonal to the scattering plane, and $L$ the distance between the multistrip detector and the sample. For the multistrip detector $H / L=0.009$ giving typically a fraction of $\sim 0.001$ grains which contribute to the diffraction profile. From Fig. 7, the total number of illuminated grains for a mean grain size of $\sim 30 \mathrm{~nm}$ is $\sim 10^{10}$, resulting in $\sim 10^{7}$ grains contributing to the spectrum for the nanocrystalline regime-a statistically significant number. 


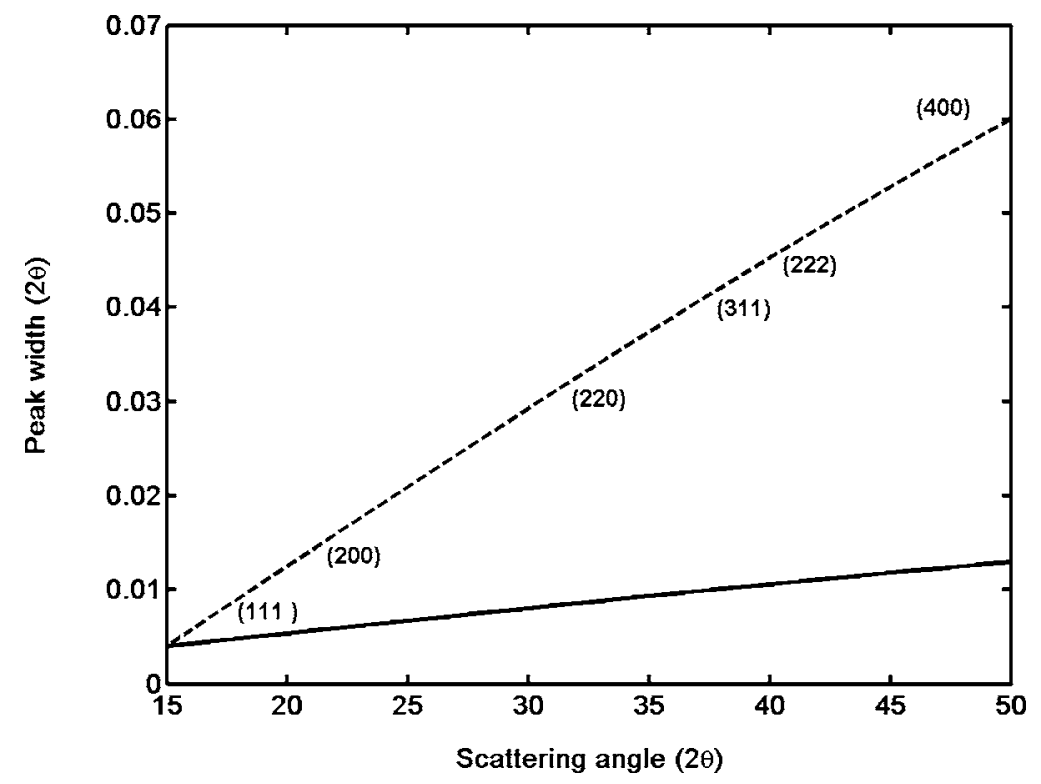

FIG. 9. Estimated width resolution for the mini-dogbones (full line) and large dog bones (dashed line). The position of the diffraction peaks of $\mathrm{Ni}$ are also indicated.

\section{B. Resolution function}

The intrinsic angular resolution of the microstrip detector is $0.004^{\circ}$. However, the resolution function is mainly determined by the sample geometry; $x$ rays that hit the sample at a different position and are diffracted under the same angle will be detected at slightly different angles in the detector. The width of the resolution function will therefore depend on the beam size, sample size, the angle between the incoming $\mathrm{x}$-ray beam and the sample, and the diffraction angle. This is demonstrated in Fig. 8. Given that the sample is illuminated completely we can estimate the width $d$ of the outgoing beam by

$$
d=W \sin (2 \theta-\alpha),
$$

where $W$ is the width of the dog bone, $2 \theta$ the diffraction angle, and $\alpha$ the angle between the sample and the direction of the incoming beam (in case the beam is smaller than the sample, $W$ becomes the beam size). As a consequence the width of the outgoing beam increases with increasing diffraction angle. This is shown in Fig. 9.

In the case of nc materials the peak width is increased due to size and strain broadening. A comparison between the width (FWHM) of the (200) diffraction peaks of coarsegrained Ni (large dog bone) and ED Ni (mini-dog-bones) is shown in Fig. 10, having, respectively, FWHM peak widths of $0.035^{\circ}$ and $0.32^{\circ}$. Also shown is ultrafine grained Ni synthesized using the high-pressure-torsion technique ${ }^{24}$ with a mean grain size of $80 \mathrm{~nm}$ (as determined by a WilliamsonHall analysis on an x-ray powder diffraction obtained with an anode source instrument), which has a FWHM peak width of $0.1^{\circ}$. Figure 10 demonstrates that in the case of high pressure torsion (HPT) and ED Ni the resolution function is at least one order of magnitude smaller than the intrinsic peak widths and therefore can be safely ignored. This is not the case for coarse-grained $\mathrm{Ni}$ (where one has to use a large dog bone, see also Sec. III A). Here the width of the resolution

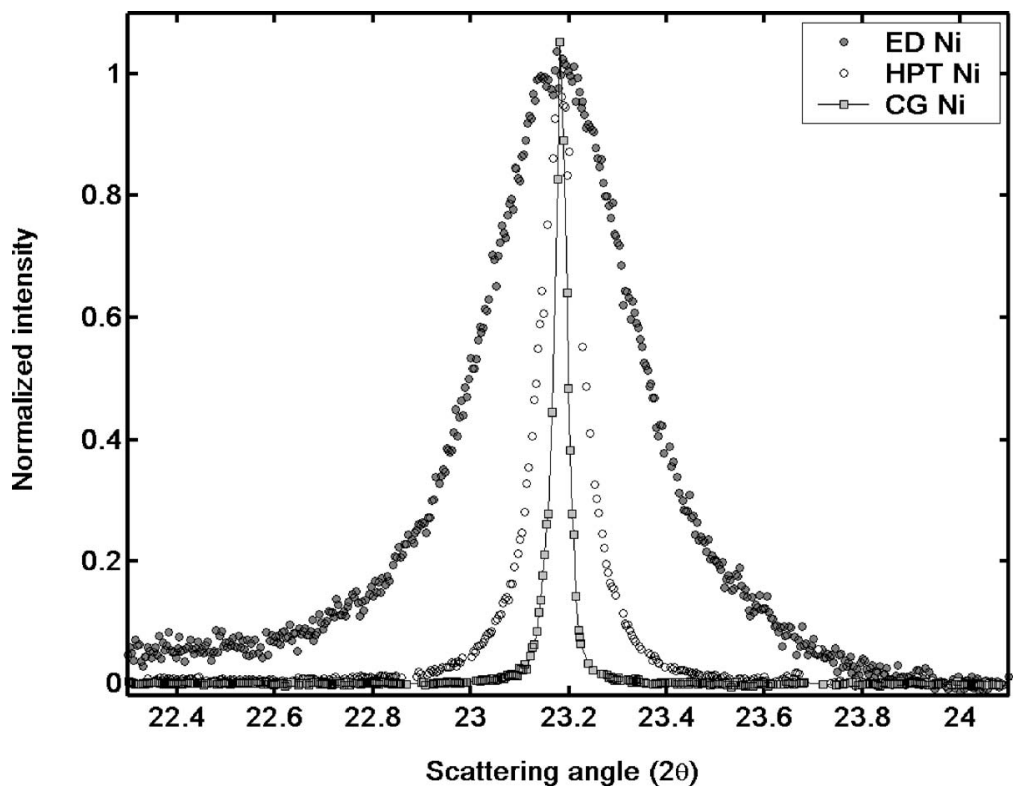

FIG. 10. (200) diffraction peak of coarse-grained HPT and ED Ni. 


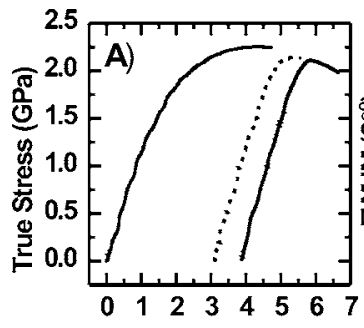

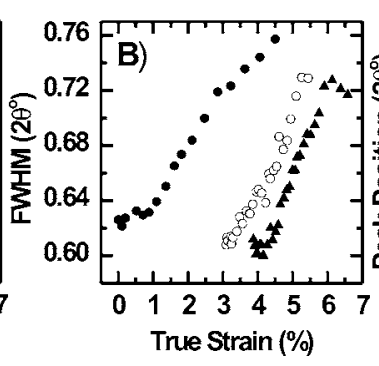

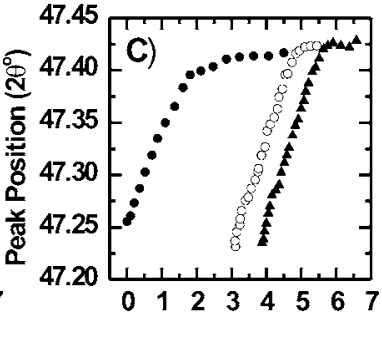

FIG. 11. (A) Stress-strain curve of three loading/unloading cycles, with corresponding (400) (B) peak width, and $(\mathrm{C})$ peak position as a function of strain. function is about half the width of the diffraction peaks. As a consequence any change of the resolution function (for instance, due to thinning of the sample during deformation) will be reflected in the peak width and has to be taken into account during data analysis.

\section{Example}

The power of this type of in situ facility is demonstrated by means of a short description of one exemplary experiment demonstrating the power of the technique to investigate the size effects coming from scaling down microstructural length scales towards the nanometer regime. The experiment demonstrates that in some nanocrystalline metals, when grain sizes are small enough and the grain-size distribution is narrow, the peak broadening observed during plastic deformation is reversible upon unloading in contradiction to what is observed for coarse-grained $\mathrm{Ni}^{23}$ A mini-dog-bone of nanocrystalline ED Ni was deformed with a constant strain rate $\left(6 \times 10^{-5}\right)$ up to a total strain of $6.5 \%$ in three subsequential loading-unloading cycles in the plastic regime. The ED Ni was purchased from Goodfellow. A Williamson-Hall analysis on the $(111) /(222)$ peaks based on data collected with a Siemens diffractometer revealed a mean grain size of $26 \mathrm{~nm}$ and $0.4 \%$ root-mean-square microstrain. The ratio of the diffraction intensities for these peaks is 2.15 , which is close to the value for a nontextured polycrystalline Ni sample (2.05). Figure 11 shows that apart from the measured stress-strain curve, also the evolution of the FWHM peak width and the peak position can be measured as function of true strain. Data are taken out of Ref. 23.

Since the (400) peak position follows a linear relation with respect to stress for the entire range of applied stress, a similar linear behavior can be expected for that part of the peak broadening coming from the inhomogeneous elastic strain, allowing the nonlinear dependence to be predominantly ascribed to plastic processes. This gives an estimate of an $\sim 50 \%$ plastic contribution to the observed peak broadening at maximum strain before unloading. In other words, upon loading the broadening is first due to elastic inhomogeneous strains up to the yield stress, where plastic deformation starts, after which the broadening comes from elastic and increasingly plastic inhomogeneous strains. A reversible peak broadening is observed for all measured diffraction peaks and demonstrates the absence of a developing residual dislocation network inside the nanosized grains, as was predicted by atomistic simulations. Such an observation would not have been possible if the peak broadening could not be followed in situ during deformation.

\section{OUTLOOK}

The in situ technique developed at the Swiss Light Source allows following the peak position and the peak broadening in situ during elastic and plastic deformation as function of applied stress and measured macroscopic strain. Due to the possibility of using different load cells and different sample grips, and additionally due to the tunability of the energy of the Swiss Light Source, there is in principle no restriction on the material that can be investigated. The current experiments are carried out in reflection, but they can also be performed in transmission especially interesting for investigating size effects in submicron thin films.

The power of this type of in situ experiment has been validated by the observation of a reversible peak broadening in nanocrystalline electrodeposited $\mathrm{Ni}$. The technique has been also recently used to study the reduction in flow stress during plastic deformation in nanocrystalline electrodeposited $\mathrm{Ni}$, demonstrating that the origin of the softening is different from the softening observed in coarse-grained $\mathrm{Ni}^{24}$ Furthermore, using low-pressure-chemical-vapor-deposited dog bones, the technique has contributed to the understanding of plastic deformation mechanism in submicron thin $\mathrm{Al}$ films. ${ }^{25}$

\section{ACKNOWLEDGMENTS}

The authors thank the Swiss National Science Foundation (Grant Nos. 2100-065151 and 200020-103714/1) for their financial support and the team of the Swiss Light Source for their technical support.

\section{APPENDIX: CALCULATION OF THE FRACTION OF ILLUMINATED GRAINS THAT CONTRIBUTE TO A BRAGG DIFFRACTION PEAK}

In the following, $\tilde{x}$ denotes the vector and $x$ its length. Consider a spherical crystallite of diameter $D$, volume $V$ $=\pi D^{3} / 6$. We can represent it by a density function

$$
\rho(\widetilde{r})=\rho(r)=1 \text { for } r \leqslant D / 2,0 \text { otherwise, }
$$

whose Fourier transform per unit volume is

$$
\begin{aligned}
F(\widetilde{q}) & =F(q)=\frac{1}{V} \int_{\mathfrak{R}^{3}} d^{3} \widetilde{r} \exp (-2 \pi i \widetilde{q} \cdot \widetilde{r}) \rho(\widetilde{r}) \\
& =3\left[\frac{\sin x-x \cos x}{x^{3}}\right]_{x=\pi q D} .
\end{aligned}
$$

The intensity of a corresponding Bragg peak centered at $\tilde{h}$ is 


$$
I_{\tilde{h}}(\widetilde{q})=C_{\tilde{h}} F^{2}(|\widetilde{q}-\widetilde{h}|),
$$

where $|\widetilde{q}-\widetilde{h}|=q+h-2 q h \cos \psi$. We may take $C_{\tilde{h}}$ such that $I_{h}(\widetilde{q})$ is normalized to 1 . Furthermore, we may approximate $I_{h}(\widetilde{q})$ by a normalized Gaussian of parameter $\sigma$ having the same FWHM,

$$
I_{\tilde{h}}(\widetilde{q})=\frac{\exp \left(-|\widetilde{q}-\widetilde{h}|^{2} / 2 \sigma^{2}\right)}{\sigma^{3}(2 \pi)^{3 / 2}} C_{\tilde{h}} F^{2}(|\widetilde{q}-\widetilde{h}|),
$$

with $\sigma=0.49 / D$.

By assuming that the $\tilde{h}$ from different grains have a uniform orientation distribution, the intensity-weighted fraction of grains scattering (just by the associated $\tilde{h}$ Bragg peak) in the volume $d^{3} \widetilde{q}$ around $\widetilde{q}$ will be

$$
\begin{aligned}
G(\widetilde{q}) d^{3} \widetilde{q}= & d^{3} \widetilde{q} \frac{1}{4 \pi} \int_{0}^{2 \pi} d \phi \int_{0}^{\pi} \\
& \times d(\cos \psi) \frac{\exp \left[-\left(q^{2}+h^{2}-2 q h \cos \psi\right) / 2 \sigma^{2}\right]}{\sigma^{3}(2 \pi)^{3 / 2}} \\
= & d^{3} \widetilde{q} \frac{\exp \left[-(q-h)^{2} / 2 \sigma^{2}\right]-\exp \left[-(q+h)^{2} / 2 \sigma^{2}\right]}{2 \sigma(2 \pi)^{3 / 2} q h} \\
& \approx d^{3} \widetilde{q} \frac{\exp \left[-(q-h)^{2} / 2 \sigma^{2}\right]}{2 \sigma(2 \pi)^{3 / 2} q h} .
\end{aligned}
$$

For the SLS Debye-Scherrer geometry, the detector pixel may be considered as a rectangle centered in the scattering plane, the center corresponding to the in-plane scattering angle of $2 \theta_{0}$, having a tangential width $w$ in the scattering plane, and height $H$ orthogonal to the scattering plane. We parametrize the pixel surface by angles $\alpha, \beta$ with

$$
-\frac{w}{2 L} \leqslant \alpha \leqslant \frac{w}{2 L}, \quad-\frac{H}{2 L} \leqslant \beta \leqslant \frac{H}{2 L},
$$

where $L$ is the sample-detector distance $(L \gg w, L \gg H)$.

We shall consider simultaneously the effects of polychromaticity and intrinsic divergence of the beam. When these effects are both very small one can assume that the beam energy is distributed in an interval

$$
\lambda_{0}\left(1-\frac{\varepsilon}{2}\right) \leqslant \lambda \leqslant \lambda_{0}\left(1+\frac{\varepsilon}{2}\right),
$$

where $0<\varepsilon \ll 1$ with uniform probability

$$
P(\lambda)=\frac{1}{\varepsilon} \text { for } \lambda_{0}\left(1-\frac{\varepsilon}{2}\right) \leqslant \lambda \leqslant \lambda_{0}\left(1+\frac{\varepsilon}{2}\right), 0 \text { otherwise. }
$$

An adimensional parameter $y \in(-\varepsilon / 2, \varepsilon / 2)$ can be used, setting $\lambda \equiv(1+y) \lambda_{0}$.

We choose a reference system so that the incident wave vector is $\widetilde{k}=(1,0,0) / \lambda$ and the scattering plane normal is $(0,0,1)$. The scattered wave vector on a point on the pixel face thus becomes

$$
\begin{aligned}
\tilde{k}^{\prime}= & \frac{1}{(1+y) \lambda_{0}}\left[\cos \left(2 \theta_{0}+\alpha\right) \cos (\beta), \sin \left(2 \theta_{0}+\alpha\right)\right. \\
& \times \cos (\beta), \sin (\beta)],
\end{aligned}
$$

and the transferred momentum

$$
\begin{aligned}
\widetilde{q}= & \widetilde{k}^{\prime}-\tilde{k}=\frac{1}{(1+y) \lambda_{0}}\left[\cos \left(2 \theta_{0}+\alpha\right) \cos (\beta)\right. \\
& \left.-1, \sin \left(2 \theta_{0}+\alpha\right) \cos (\beta), \sin (\beta)\right],
\end{aligned}
$$

having a norm (to first order in $\alpha, \beta, y$ ) equal to

$$
q \approx \frac{2 \sin \left(\theta_{0}\right)(1-y)+\alpha \cos \left(\theta_{0}\right)}{\lambda_{0}} .
$$

Taking the first-order expansion of the Jacobian, we can write

$$
\begin{aligned}
d^{3} \widetilde{q} & \equiv J_{\widetilde{q} ; \alpha, \beta, y} d \alpha d \beta d y \\
& \approx-2 \sin \left(\theta_{0}\right) d \alpha d \beta d y \frac{(1-4 y) \sin \left(\theta_{0}\right)+\alpha \cos \left(\theta_{0}\right)}{\lambda_{0}^{3}} .
\end{aligned}
$$

To evaluate the intensity-weighted number fraction of grains which will contribute, by their associated Bragg peak $\widetilde{h}$, to the signal on the considered pixel centered at $2 \theta_{0}$, we must evaluate

$$
\begin{aligned}
{\left[\frac{d N}{N}\right]_{2 \theta_{0}} } & \\
& =\lim _{\varepsilon \rightarrow 0^{+}} \int_{-w / 2 L}^{w / 2 L} d \alpha \int_{-H / 2 L}^{H / 2 L} d \beta \int_{-\varepsilon / 2}^{\varepsilon / 2} d y J_{\tilde{q} ; \alpha, \beta, y} \frac{1}{\varepsilon} G(q),
\end{aligned}
$$

where $G(q)=G(\widetilde{q})$ is given in Eq. (A1) with $q$ from Eq. (A2) and $J_{\widetilde{q} ; \alpha, \beta, y}$ from Eq. (A3). The limit on $\varepsilon \rightarrow 0^{+}$follows because we are interested in the case of negligible divergence and polychromaticity. The result, to first order in $H / L, w / L$, after setting $q_{0}=2 \sin \left(\theta_{0}\right) / \lambda_{0}$ and using $\sigma=0.49 / D$, is

$$
\begin{aligned}
{\left[\frac{d N}{N}\right]_{2 \theta_{0}} } & =\frac{q_{0}}{h} \frac{w H}{L^{2}} \frac{D}{0.49 \lambda_{0}} \frac{\exp \left[-1.041\left(q_{0}-h\right)^{2} D^{2}\right]}{2(2 \pi)^{3 / 2}} \\
& =0.0648 \frac{q_{0}}{h} \frac{w H}{L^{2}} \frac{D}{\lambda_{0}} \exp \left[-1.041\left(q_{0}-h\right)^{2} D^{2}\right] .
\end{aligned}
$$

In the above we have considered only a single Bragg peak. To keep in consideration all symmetry-equivalent Bragg peaks, we need to insert an additional factor $p$, which is the multiplicity of the considered Bragg reflection under the action of the Laue group.

To obtain the fraction of grains contributing to the whole powder profile of the Bragg peak $\tilde{h}$, we have to sum the former expression over all pixels in the whole angular range. To approximate the sum by an integral, we divide by the pixel angular width $w / L$ and integrate with respect to $\theta_{0}$ $=\arcsin \left(q_{0} \lambda_{0} / 2\right)$, giving finally

$$
\begin{aligned}
{\left[\frac{d N}{N}\right]_{h} } & \approx \frac{p}{w / L} \int_{\text {allpeak }} d \theta_{0}\left[\frac{d N}{N}\right]_{2 \theta_{0}} \\
& \approx \frac{p}{w / L} \int_{0}^{+\infty} d q_{0} \frac{\lambda_{0}}{\sqrt{4-q_{0}^{2} \lambda_{0}^{2}}}\left[\frac{d N}{N}\right]_{2 \theta_{0}} \\
& \approx \frac{0.028 p H / L}{\cos \left(\theta_{h}\right)}\left\{1+O\left[(\lambda / D)^{2}\right]\right\}
\end{aligned}
$$


where $\theta_{h}=\arcsin \left(h_{0} \lambda_{0} / 2\right)$ which is the diffraction half-angle corresponding to the center of the peak.

${ }^{1}$ J. R. Weertman, Nanostructured Materials: Processing, Properties, and Potential Applications (William Andrew, Norwich, NY, 2002), Chap. 10.

${ }^{2}$ K. S. Kumar, H. Van Swygenhoven, and S. Suresh, Acta Mater. 51, 5743 (2003).

${ }^{3}$ M. D. Uchic, D. M. Dimiduk, J. N. Florando, and W. D. Nix, Science 305, 986 (2004).

${ }^{4}$ H. Van Swygenhoven, Science 296, 66 (2002).

${ }^{5}$ H. Van Swygenhoven, P. M. Derlet, and A. Froseth, Nat. Mater. 3, 399 (2004).

${ }^{6}$ K. S. Kumar, S. Suresh, M. F. Chisholm, J. A. Horton, and P. Wang, Acta Mater. 51, 387 (2003)

${ }^{7}$ Diffraction Analysis of the Microstructure of Materials, Springer Series in Materials Science 68 edited by E. J. Mittemeijer and P. Scardi (Springer, New York, 2003).

${ }^{8}$ R. Yazici, W. Mayo, T. Takemoto, and S. Weissmann, J. Appl. Crystallogr. 16, 89 (1983).

${ }^{9}$ H. Y. Liu, W. E. Mayo, and S. Weissmann, Mater. Sci. Eng. 63, 81 (1984).

${ }^{10}$ J. Böhm, P. Gruber, R. Spolenak, A. Stierle, A. Wanner, and E. Arzt, Rev. Sci. Instrum. 75, 1110 (2004).

${ }^{11}$ R. Spolenak et al., Phys. Rev. Lett. 90, 096102 (2003).
${ }^{12}$ B. Schmitt et al., Nucl. Instrum. Methods Phys. Res. A 518, 436 (2004).

${ }^{13}$ B. D. Patterson et al., Nucl. Instrum. Methods Phys. Res. A 540, 42 (2005).

${ }^{14}$ W. S. Sharpe, Jr., B. Yuan, and R. L. Edwards, J. Microelectromech. Syst. 6, 193 (1997).

${ }^{15}$ D. Gianola, K. Hemker, M. Legros, and W. Sharpe, Jr., TMS Lett. 1, 149 (2004).

${ }^{16}$ H. Toraya, J. Appl. Crystallogr. 19, 440 (1986).

${ }^{17}$ P. J. Huber, Robust Statistics (Wiley, New York, 1981).

${ }^{18}$ A. Cervellino, P. M. Derlet, and H. Van Swygenhoven, Acta Mater. (2006) (in press).

${ }^{19} \mathrm{C}$. Genzel, in Diffraction Analysis of the Microstructure of Materials, edited by E. J. Mittemeijer and P. Scardi (Springer-Verlag, Berlin, 2004), pp. 373-503.

${ }^{20}$ Source: http://physics.nist.gov/PhysRefData/XrayMassCoef/ElemTab/ z28.html

${ }^{21}$ J. Ladell, Acta Crystallogr. 14, 47 (1961).

${ }^{22}$ I. C. Noyan and S. K. Kaldor, Powder Diffr. 19, 104 (2004).

${ }^{23}$ Z. Budrovic, H. Van Swygenhoven, P. M. Derlet, S. Van Petegem, and B. Schmitt, Science 304, 273 (2004).

${ }^{24}$ Z. Budrovic, S. Van Petegem, P. M. Derlet, B. Schmitt, and H. Van Swygenhoven, Appl. Phys. Lett. 86, 231910 (2005).

${ }^{25}$ D. Gianola, S. Van Petegem, M. Legros, S. Brandstetter, H. Van Swygenhoven, and K. Hemker (unpublished). 